01,02

\title{
Импульсное возбуждение в двухкубитных системах
}

\author{
(C) Я.С. Гринберг, А.А. Штыгашев \\ Новосибирский государственный технический университет, \\ Новосибирск, Россия \\ E-mail: yakovgreenberg@yahoo.com
}

\begin{abstract}
Исследована временна́я динамика поглощения однофотонного импульса двумя кубитами, взаимодействующими с микроволновым полем одномерного волновода. Разработана теория, которая позволяет в качестве начального условия использовать любые формы входного однофотонного волнового пакета, а также исследовать динамику возбуждения каждого кубита. Численный расчет проведен для пакета гауссовой формы при разных параметрах частотной расстройки и длительности входного импульса. Исследована динамика возбуждения как идентичных, так и неидентичных кубитов. Показано, в частности, что для идентичных кубитов возможно формирование симметричных и антисимметричных запутанных состояний.
\end{abstract}

Работа выполнена при финансовой поддержке Российского научного фонда, грант № 16-19-10069.

DOI: 10.21883/FTT.2018.11.46641.02NN

\section{1. Введение}

Большинство протоколов записи и считывания информации в кубитных системах основано на последовательности возбуждающих и считывающих импульсов. В ряде работ исследовалась вероятность возбуждения однофотонным импульсом одного кубита [1-6]. В этих работах показано, что максимальная вероятность возбуждения кубита зависит от фотонного состояния (когерентного или фоковского) а также от формы импульса [1]. Идеальное инвертирование кубита с вероятностью 100\%, отвечающее полному поглощению падающего импульса в некоторый момент времени, достигается только с помощью импульса специальной формы $[2,3]$.

Фотонный импульс можно разбить на четную и нечетную компоненту. С кубитом взаимодействует только четная компонента [3], поэтому, если фотон падает с одного направления, то кубит может поглотить только 50\% энергии импульса. При этом максимальная вероятность возбуждения кубита не может быть больше $50 \%$.

Численные расчеты показывают, что вероятность возбуждения кубита в одномерном открытом волноводе однофотонным гауссовым импульсом спектральная ширина которого порядка скорости спонтанного излучения не превышает 40\% [4].

В работах [7-9] рассматривалось импульсное возбуждение двухатомных $[7,8]$ и многоатомных [9] двухуровневых систем, состоящих из одинаковых атомов с одной и той же частотой возбуждения. В отличие от реальных атомов искусственные атомы, к которым, в частности, относятся твердотельные кубиты, могут иметь технологический разброс по своим параметрам и, кроме того, они могут быть целенаправленно изготовлены неидентичными для конкретных применений. Поэтому, в настоящей работе в отличие от работ [7-9], исследован также процесс импульсного возбуждения двух кубитов, неидентичность которых с самого начала заложена в исходные динамические уравнения. Основное внимание мы уделили динамическому поведению вероятности возбуждения каждого из кубитов.

В рамках марковского приближения обнаружены некоторые новые особенности динамического поведения амплитуд вероятности при возбуждении идентичных кубитов. В частности, при импульсном возбуждении двух кубитов возможно формирование симметричных и антисимметричных запутанных состояний. Обнаружено также, что фазы амплитуд возбуждения кубитов, каждый из которых находится в резонансе с центральной частотой волнового пакета не зависит от времени. Это свойство сохраняется при произвольном расстоянии между кубитами.

Статья организована следующим образом. В разделе 2 приводится краткий вывод основных динамических уравнений, описывающих динамическое поведение амплитуд возбуждений каждого из кубитов. В первой части раздела 3 исследуется процесс возбуждения двух идентичных кубитов. Во второй части раздела 3 рассматриваются особенности возбуждения неидентичных кубитов.

\section{2. Постановка задачи}

Гамильтониан системы включает три элемента (здесь и далее $\hbar=1)$, описывающих, соответственно, два кубита $\left(0.5 \Omega_{1} \sigma_{z}^{(1)}+0.5 \Omega_{2} \sigma_{z}^{(2)}\right)$, гамильтониан электромагнитного поля в волноводе $\left(\sum_{k} \omega_{k} a_{k}^{(+)} a_{k}\right)$ и взаимодействие кубитов с электромагнитным полем волновода.

$$
H_{\text {int }} \sum_{k}\left(g_{k}^{(1)} e^{-i k x_{1}} \sigma^{(1)}+g_{k}^{(2)} e^{-i k x_{2}} \sigma^{(2)}\right) a_{k}^{+}+\text {c.c. }
$$

Кубиты фиксированы в точках $x_{1}, x_{2}$ на расстоянии $d$ друг от друга $\left(x_{1}=-d / 2, x_{2}=+d / 2\right)$. Величины $g_{k}^{(i)}$ представляют собой константы взаимодействия $i$-го кубита с фотонным полем резонатора. Волновую функцию системы запишем в однофотонном приближении в виде 
суперпозиции трех состояний $\left|e_{1} g_{2} 0\right\rangle,\left|g_{1} e_{2} 0\right\rangle,\left|g_{1} g_{2} k\right\rangle$, где в первых двух один из кубитов находится в возбужденном состоянии $|e\rangle$, а другой в основном состоянии $|g\rangle$, и при этом фотон в волноводе отсутствует. В третьем состоянии $\left|g_{1} g_{2} k\right\rangle$ оба кубита находятся в основном состоянии и в волноводе имеется один фотон.

$$
\begin{aligned}
|\Psi\rangle= & \beta_{1}(t) e^{-i \Omega_{1} t}\left|e_{1} g_{2} 0\right\rangle+\beta_{2}(t) e^{-i \Omega_{2} t}\left|g_{1} e_{2} 0\right\rangle \\
& +\sum_{k} \gamma_{k}(t) e^{-i \omega_{k} t}\left|g_{1} g_{2} k\right\rangle,
\end{aligned}
$$

Амплитуды возбуждения кубитов $\beta_{1}$ и $\beta_{2}$ и фотонного импульса $\gamma_{k}$ определяются из уравнения Шредингера $i d|\Psi\rangle / d t=H|\Psi\rangle$

$$
\begin{aligned}
\frac{d \beta_{j}}{d t} & =-i \sum_{k} g_{k}^{(j)} \gamma_{k}(t) e^{i k x j} e^{-i\left(\omega_{k}-\Omega_{j}\right) t}, \quad(j=1,3), \\
\frac{d \gamma_{k}}{d t} & =-i g_{k}^{(1)^{*}} \beta_{1}(t) e^{-i k x_{1}} e^{i\left(\omega_{k}-\Omega_{1}\right) t} \\
& -i g_{k}^{2^{*}} \beta_{2}(t) e^{-i k x_{2}} e^{i\left(\omega_{k}-\Omega_{2}\right) t} .
\end{aligned}
$$

Суммирование по $k$ в (3) идет по всем непрерывным значениям $k$, положительным и отрицательным, с учетом того что частота $\omega_{k}$ связана только с абсолютным значением $k: \omega_{k}=v_{g}|k|$. В дальнейшем при выводе уравнений (6) переход от суммирования к интегрированию осуществляется согласно следующему выражению:

$$
\sum_{k} \Longrightarrow 2 \frac{L}{2 \pi} \int_{-\infty}^{\infty} d k=2 \frac{L}{2 \pi} 2 \int_{0}^{\infty} d|k|=2 \frac{L}{\pi v_{g}} \int_{0}^{\infty} d \omega_{k},
$$

где множитель 2 в первом члене справа учитывает две независимые поляризации фотона, а $L / 2 \pi-$ плотность состояний фотонных мод в одномерном открытом волноводе.

Формальное решение уравнения (4) есть

$$
\begin{aligned}
\gamma_{k}(t)= & \gamma_{k}(0)-i g_{k}^{(1)^{*}} e^{-i k x_{1}} \int_{0}^{t} \beta_{1}\left(t^{\prime}\right) e^{i\left(\omega_{k}-\Omega_{1}\right) t^{\prime}} d t^{\prime} \\
& -i g_{k}^{(2)^{*}} e^{-i k x_{2}} \int_{0}^{t} \beta_{2}\left(t^{\prime}\right) e^{i\left(\omega_{k}-\Omega_{2}\right) t^{\prime}} d t^{\prime},
\end{aligned}
$$

где величина $\gamma_{k}(0)$ в (5) представляет собой волновой пакет в $k$-пространстве в начальный момент времени.

Подставив это решение в уравнение (3), получим в приближении Вайскопфа-Вигнера [10], уравнения для амплитуд вероятности $\beta_{1}, \beta_{2}$

$$
\begin{gathered}
\frac{d \beta_{m}}{d t}=-i \sqrt{\frac{\Gamma_{m} v_{g}}{4 \pi}}\left(\frac{\omega_{s}}{\Omega_{m}}\right)^{1 / 2} \int_{-\infty}^{\infty} \gamma_{k}(0) e^{i k x_{m}} e^{-i\left(\omega_{k}-\Omega_{m}\right) t} d k \\
-\frac{\Gamma_{m}}{2} \beta_{m}(t)-\frac{\sqrt{\Gamma_{m} \Gamma_{n}}}{2}\left(\frac{\Omega_{n}}{\Omega_{m}}\right)^{1 / 2} e^{i k_{n}\left(x_{m}-x_{n}\right)} e^{i\left(\Omega_{m}-\Omega_{n}\right) t} \beta_{n}(t),
\end{gathered}
$$

где $m, n=1,2$ и в каждом уравнении $m \neq n$, $k_{m}=\Omega_{m} / v_{g}, v_{g}$ - групповая скорость микроволнового фотона в волноводе, которую в последующих расчетах мы приняли равной скорости света в пустом пространстве.

Величины $\Gamma_{m}$, представляют собой скорости спонтанного излучения соответствующего кубита, которые в приближении Вайскопфа-Вигнера выражаются через параметры взаимодействия $g_{k}^{(i)}: \Gamma_{t}=4 L\left|g_{\Omega}^{(i)}\right|^{2} / v_{g}$, где

$$
\left|g_{\Omega}^{(i)}\right|^{2}=\frac{1}{2}\left(\frac{D^{2} \Omega_{i}}{2 \hbar \varepsilon_{0} V}\right)
$$

В выражении (7) $D$ - это дипольный момент кубита, $V$ - эффективный объем взаимодействия кубита с электромагнитным полем в волноводе. Заметим, что величины $\Gamma_{i}$ и $g_{k}^{(i)}$ рассчитать теоретически довольно сложно. Поэтому ниже в качестве величин $\Gamma_{i}$ мы будем брать их экспериментальные значения, характерные для сверхпроводникового потокового кубита.

В качестве исходного волнового пакета в $k$-пространстве мы взяли гауссов пакет

$$
\gamma_{k}^{(0)}=\left(\frac{2}{\pi \Delta^{2}}\right)^{1 / 4} \exp \left(i\left(k-k_{s}\right) x_{0}-\frac{\left(k-k_{s}\right)^{2}}{\Delta^{2}}\right),
$$

где $\Delta-$ спектральная ширина пакета в $k$-пространстве, связанная с пространственной шириной пакета: $\sigma=\sqrt{2} / \Delta,-x_{0}-$ положение максимума огибающей на оси $x$ в начальный момент времени, $k_{s}=\omega_{s} / v_{g}$, где $\omega_{s}$ - частота центра фотонного импульса.

Волновой пакет (8) нормирован на единицу: $\int_{-\infty}^{\infty}\left|\gamma_{k}(0)\right|^{2} d k=1$. В координатном пространстве распределению (8) до рассеяния соответствует волновой пакет с гауссовой огибающей, распространяющийся слева направо:

$$
\begin{aligned}
u(x, t) & =\frac{1}{\sqrt{2 \pi}} \int_{-\infty}^{\infty} \gamma_{k}^{(0)} e^{-i k\left(x-v_{g} t\right)} d k \\
& =\left(\frac{\Delta^{2}}{2 \pi}\right)^{1 / 4} e^{-\Delta^{2}\left(x+x_{0}-v_{g} t\right)^{2} / 4} e^{-i k_{s}\left(x-v_{g} t\right)} .
\end{aligned}
$$

\section{3. Временна́я динамика амплитуд возбуждения кубитов}

\section{1. Идентичные кубиты}

Ниже приведены результаты численного расчета временно́й динамики амплитуд возбуждения кубитов $\beta_{1}(t)$ и $\beta_{2}(t)$, описываемых уравнениями (6) для идентичных кубитов $\left(\Omega_{1}=\Omega_{2}=\Omega, \Gamma_{1}=\Gamma_{2}=\Gamma\right)$. Исходные параметры пакета $(8): \Delta=0.033 \mathrm{~m}^{-1}$, что соответствует $\sigma \cong 46 \mathrm{~m}$ и спектральной ширине пакета $\Delta \omega / 2 \pi=\Delta v_{g}=1.6 \mathrm{MHz} ; x_{0}=300 \mathrm{~m}$. В качестве численных значений параметров кубитов были взяты значения, характерные для сверхпроводникового потокового кубита $[11,12]: \Omega / 2 \pi=5 \mathrm{GHz}\left(\lambda=2 \pi v_{g} / \Omega=6 \mathrm{~cm}\right)$, 


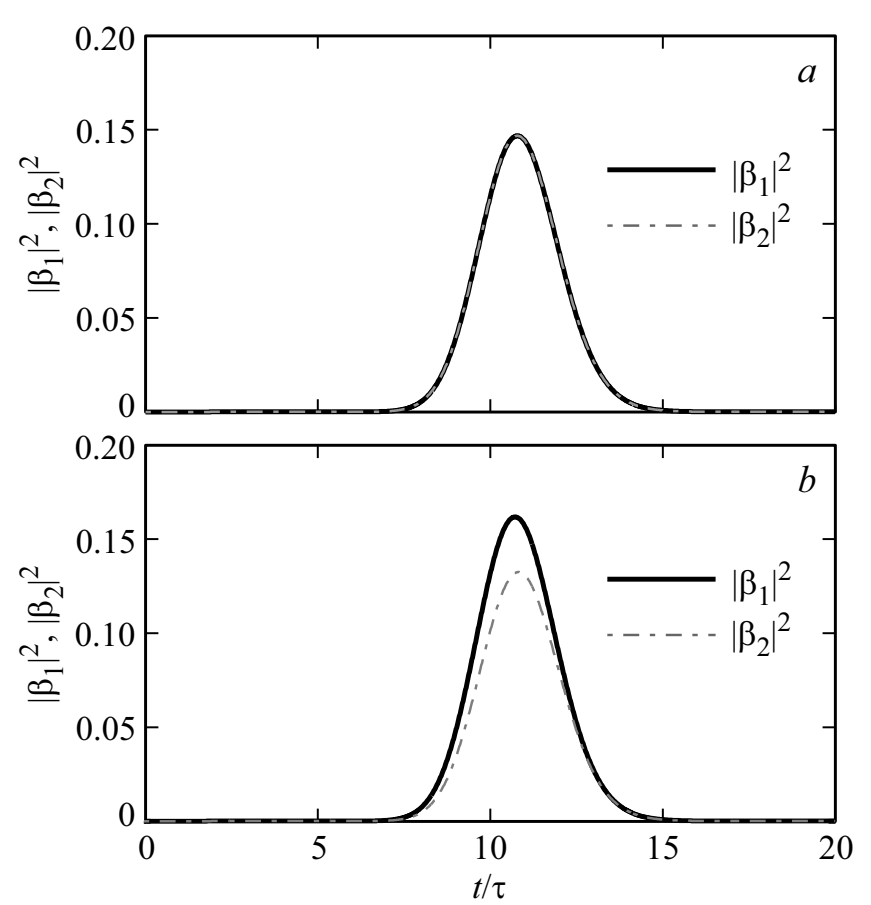

Рис. 1. Зависимость от времени вероятности возбуждения двух идентичных кубитов однофотонным импульсом. $\Omega / 2 \pi=\omega_{s} / 2 \pi=5 \mathrm{GHz}, \Gamma / 2 \pi=1.6 \mathrm{MHz} . a-k d=0.033 \pi$, $d=1 \mathrm{~mm} ; b-k d=100 \pi, d=3 \mathrm{~m}$.

$\Gamma / 2 \pi=1.6 \mathrm{MHz}$ при различных значениях величины $k d=\left(\Omega / v_{g}\right) d$, где $d-$ расстояние между кубитами. На всех прилагаемых ниже рисунках временно́й масштаб $\tau=10^{-7} \mathrm{~s}$.

На рис. 1 показана временна́я динамика вероятности возбуждения двух идентичных кубитов $\left|\beta_{1}(t)\right|^{2},\left|\beta_{2}(t)\right|^{2}$, находящихся в резонансе с частотой налетающего фотона $\Omega=\omega_{s}$ при разных расстояниях $d$ между кубитами. Если расстояние между кубитами $d$ много меньше длины волны $\lambda$, соответствующей частоте центра пакета $\omega_{s}$, то вероятности возбуждения двух кубитов практически одинаковы (рис. 1,a). В случае, когда расстояние между кубитами $d \gg \lambda$ вероятность возбуждения первого кубита (фотон падает слева) несколько больше (рис. $1, b$ ) и опережает по времени процесс возбуждения второго кубита.

Известно, что при фиксированной форме огибающей пакета вероятность возбуждения кубита зависит от отношения спектральной ширины пакета $\Delta v_{g}$ к скорости спонтанного излучения $\Gamma[1]$. На рис. 2 такая зависимость показана при возбуждении двух одинаковых кубитов гауссовым импульсом. Из этого рисунка видно, что вероятность резонансного возбуждения каждого из двух идентичных кубитов гауссовым пакетом не превышает 20\%. Функционально эта зависимость совпадает по форме с аналогичной кривой, полученной в [1] при возбуждении одного кубита. На начальном участке $\left(\Delta v_{g} / \Gamma<1\right)$, пока спектральная ширина пакета $\Delta v_{g}$ мала, узкий острый пик гауссового распределения вблизи его центра приводит к тому, что вероятность возбуждения пропорциональна ширине пакета $\Delta v_{g}$. При $\Delta v_{g} / \Gamma \gg 1$ эффективность резонансного поглощения снижается за счет широкой полосы импульса и, соответственно, уменьшения плотности энергии импульса. Таким образом, конкуренция этих двух факторов приводит к появлению максимума при некотором промежуточном значении величины $\Delta v_{g} / \Gamma$. Как следует из рис. 2 , максимальная амплитуда достигается при $\Delta v_{g} / \Gamma \approx 3$. Как мы увидим ниже, приведенное выше качественное объяснение в применении к возбуждению двух кубитов является, по-видимому, сильно упрощенным.

Импульсное возбуждение двух кубитов позволяет сформировать симметричные и антисимметричные запутанные состояния. Первые два слагаемых в выражении (2) в общем случае (при произвольных коэффициентах $\left.\beta_{i}\right)$ представляют собой запутанное состояние двух кубитов. Если расстояние между кубитами меньше или сравнимо с длиной волны, то возможно формирование запутанных симметричных и антисимметричных состояний. Из рис. 3 видно, что запутанное антисимметричное состояние $\operatorname{Re} \beta(t)\left(\left|g_{1} e_{2}\right\rangle-\left|e_{1} g_{2}\right\rangle\right)$ формируется при $k d=(2 n+1) \pi, n=0,1,2 \ldots$ когда $\operatorname{Im} \beta_{1}=\operatorname{Im} \beta_{2}=0$, $\operatorname{Re} \beta_{1}=-\operatorname{Re} \beta_{2}$ (рис. $\left.3, a\right)$, тогда как запутанное симметричное состояние $i \operatorname{Im} \beta(t)\left(\left|g_{1} e_{2}\right\rangle+\left|e_{1} g_{2}\right\rangle\right)$ формируется при $k d=2 n \pi, n=0,1,2, \ldots$, когда $\operatorname{Im} \beta_{1}=\operatorname{Im} \beta_{2}$, $\operatorname{Re} \beta_{1}=\operatorname{Re} \beta_{2}=0$ (рис. $\left.3, b\right)$.

Как известно, для описания степени запутанности состояний в квантовой механике вводится некая величина $C(t)$, которая применительно к нашей системе, описываемой волновой функцией (2), имеет вид [9]

$$
C(t)=\max \left\{0, \sqrt{\left|\beta_{1}(t)\right|\left|\beta_{2}(t)\right|}-\sqrt{2}\left|\beta_{1}(t)\right|\left|\beta_{2}(t)\right|\right\} .
$$

Следует отметить, что эта величина определяет степень запутанности двух кубитов, взаимодействующих через общее электромагнитное поле в волноводе. В этом

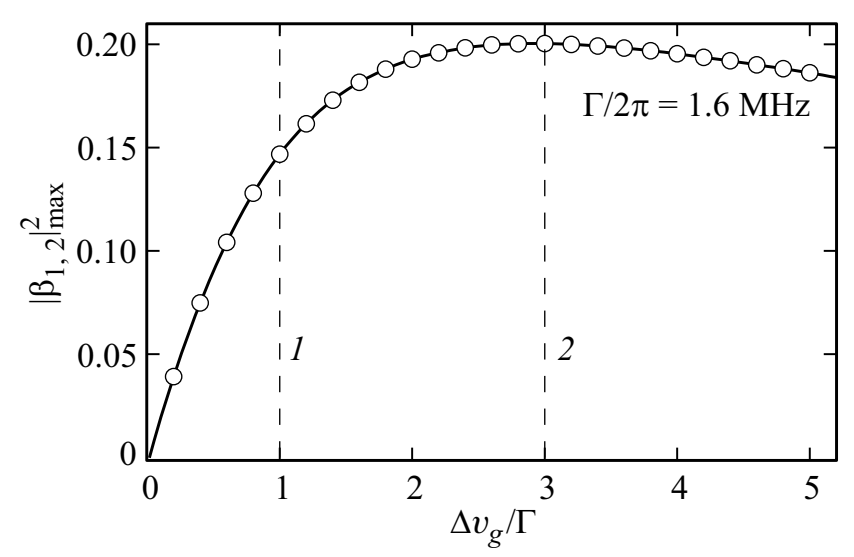

Рис. 2. Зависимость максимальных амплитуд вероятности возбуждения двух идентичных кубитов от отношения $\Delta v_{g} / \Gamma$. Параметры: $k d=0.033 \pi, d=1 \mathrm{~mm}, \Omega / 2 \pi=\omega_{s} / 2 \pi=5 \mathrm{GHz}$, $\Gamma / 2 \pi=1.6 \mathrm{MHz}$. Расчет проводился при фиксированной величине $Г$. 


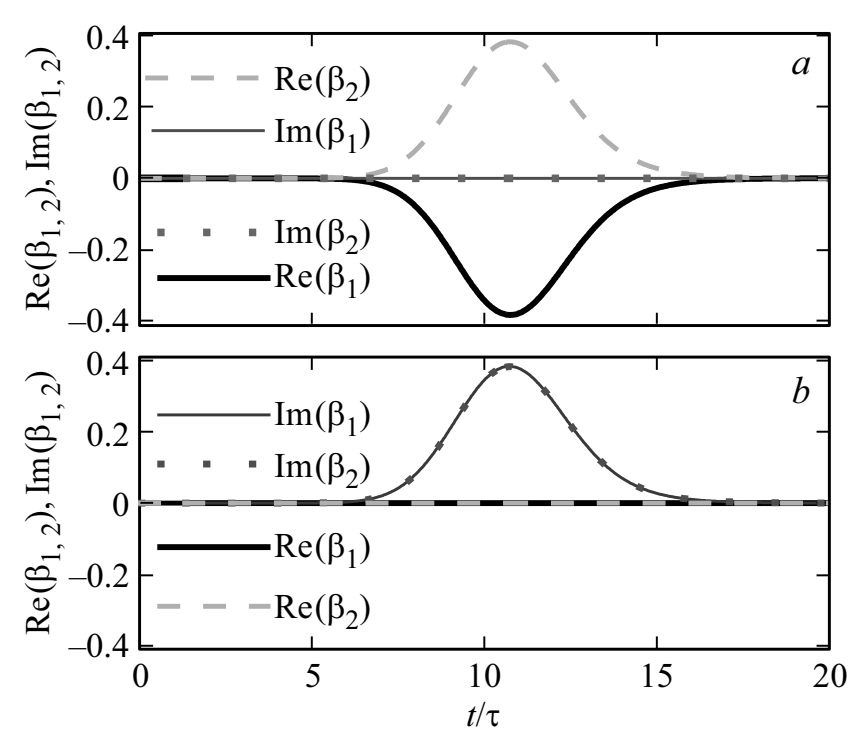

Рис. 3. Зависимость от времени вещественной и мнимой частей амплитуд вероятности резонансного возбуждения идентичных кубитов. $\Omega / 2 \pi=\omega_{s} / 2 \pi=5 \mathrm{GHz}, \Gamma / 2 \pi=1.6 \mathrm{MHz}$; $a-k d=\pi, d=0.03 \mathrm{~m} ; b-k d=2 \pi, d=0.06 \mathrm{~m}$.

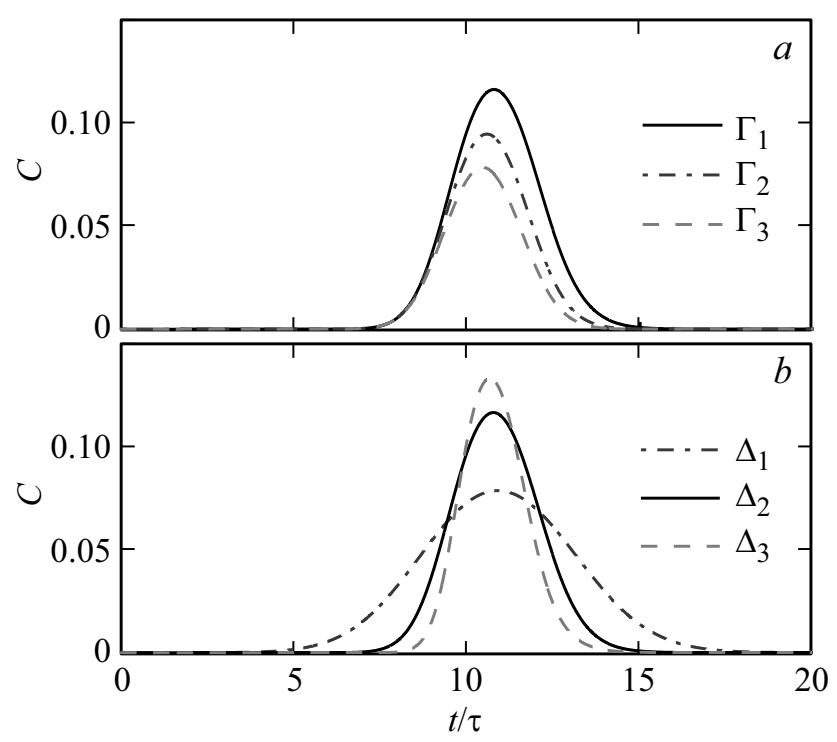

Рис. 4. Зависимость от времени степени запутанности при резонансном возбуждении двух идентичных кубитов. $\Omega / 2 \pi=\omega_{s} / 2 \pi=5 \mathrm{GHz}, k d=\pi, d=0.03 \mathrm{~m} . a-\Delta=\Gamma_{1} / v_{g}$, $\Gamma_{1} / 2 \pi=1.6 \mathrm{MHz}, \quad \Gamma_{2} / 2 \pi=2.4 \mathrm{MHz}, \quad \Gamma_{3} / 2 \pi=3.2 \mathrm{MHz}$. $b-\Gamma / 2 \pi=1.6 \mathrm{MHz}, \Delta_{1}=0.5 \Gamma / v_{g}, \Delta_{2}=\Gamma / v_{g}, \Delta_{3}=1.5 \Gamma / v_{g}$.

смысле выражение (10) существенно отличается от аналогичного выражения, описывающего степень запутанности состояний двух кубитов с прямым дипольдипольным взаимодействием. В частности, простой анализ выражения (10) показывает, что $C(t)$ достигает максимума, равного $\sqrt{2} / 8$, при $\left|\beta_{1}\right|=\left|\beta_{2}\right|=\sqrt{2} / 4$, тогда как при $\left|\beta_{1}\right|=\left|\beta_{2}\right|=1 / \sqrt{2}$ величина $C(t)=0$. На рис $4, a$ показана зависимость $C(t)$ для запутанного состояния, изображенного на рис. 3, $a$, для трех пар идентичных кубитов, при различных значениях скоростей спонтанной эмиссии для каждой пары. Наши расчеты, проведенные в широком диапазоне параметра $d(d \leq 15 \lambda)$, показывают, что при резонансном возбуждении двух кубитов с одинаковыми частотами зависимость $C(t)$ от времени имеет практически (и даже численно) один и тот же вид, аналогичный показанному на рис. $4, a$, Из этого следует, что параметр степени запутанности $C(t)$ при импульсном возбуждении двух идентичных кубитов не несет в целом определенной информации о расстоянии между кубитами. Тем не менее, на форму линии $C(t)$ существенно влияет спектральная ширина импульса $\Delta$ (рис. $4, b$ ). Чем меньше спектральная ширина пакета, тем больше его длительность во времени и тем дольше он взаимодействует с кубитами. При этом ширина линии $C(t)$ естественно возрастает. И наоборот, при увеличении $\Delta$ пакет во временно́й области становится уже и длительность его взаимодействия с кубитами уменьшается, что приводит к сужению линии $C(t)$.

Мы также проанализировали временну́ю зависимость главных значений фаз амплитуд $\beta_{i}(i=1,2)$ : $\alpha_{i}=\arctan \left(\operatorname{Im} \beta_{i} / \operatorname{Re} \beta_{i}\right) \quad\left(-\pi / 2<\alpha_{i}<\pi / 2\right)$. Мы ожидали, что каждая из фаз $\alpha_{i}$ будет зависеть от времени, тогда как их разность будет постоянной. Оказалось, однако, что для идентичных кубитов в случае резонансного возбуждения $\left(\omega_{s}=\Omega\right)$ фазы $\alpha_{i}$ не зависят от времени.

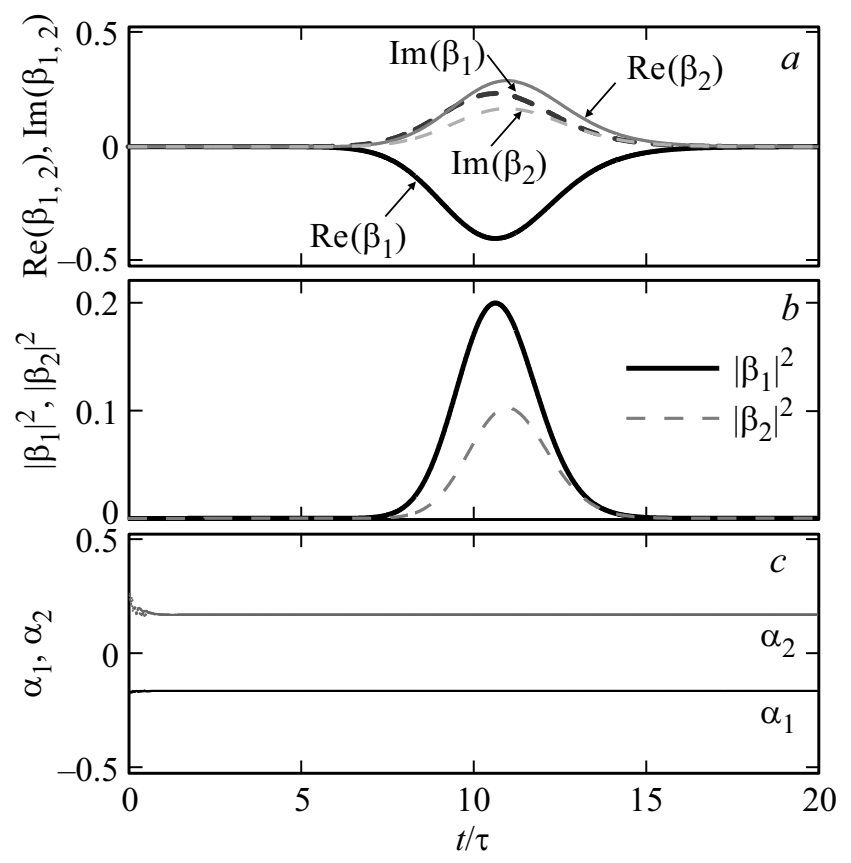

Рис. 5. Импульсное возбуждение двух идентичных кубитов. $k d / \pi=333.333, d=10 \mathrm{~m}, \Omega / 2 \pi=\omega_{s} / 2 \pi=5 \mathrm{GHz}$, $\Gamma / 2 \pi=1.6 \mathrm{MHz} . a-$ зависимость от времени вещественной и мнимой частей амплитуд вероятности. $\operatorname{Re} \beta_{1}$ и $\operatorname{Im} \beta_{1}$ показаны жирными сплошной черной и штриховой синей линиями соответственно. $\operatorname{Re} \beta_{2}$ и $\operatorname{Im} \beta_{2}$ показаны тонкими сплошной и штриховой линиями соответственно. $b-$ зависимость вероятности возбуждения двух кубитов от времени. $c$ - временна́я зависимость фаз амплитуд возбуждения двух кубитов, $\alpha_{2}-\alpha_{1}=0.333$. 
Это свойство продемонстрировано на рис. 5, где мы намеренно взяли большое расстояние между кубитами $(d=10 \mathrm{~m})$, так что возбуждение второго кубита заметно запаздывает (рис. $5, b)$, величины $\operatorname{Re} \beta_{i}, \operatorname{Im} \beta_{i}$ зависят от времени (рис. 5, $a$ ), а фазы $\alpha_{i}$ от времени не зависят (рис. 5,c). При этом разность фаз $\alpha_{1}-\alpha_{2}$ тоже постоянна и равна по величине дробной части числа $k d / \pi$. Это свойство характерно только для резонансного возбуждения. Если же $\omega_{s} \neq \Omega$, то каждая из фаз $\alpha_{i}$ зависит от времени, хотя разность между ними, как и следует ожидать, остается постоянной и по-прежнему равна по величине дробной части числа $k d / \pi$.

\section{2. Неидентичные кубиты}

Если частоты возбуждения кубитов неодинаковы $\left(\Omega_{1} \neq \Omega_{2}\right)$, то наличие последнего слагаемого в правой части уравнения (6) приводит к осцилляторной зависимости от времени величин $\beta_{1}$ и $\beta_{2}$. При этом возбуждается в основном тот кубит, частота которого совпадает с центральной частотой пакета $\omega_{s}$ (рис. $\left.6, c\right)$. В этом случае достигается максимальная вероятность возбуждения резонансного кубита, равная 40\%, что согласуется с результатом, полученным в [4]. При этом реальная и мнимая части амплитуды резонансного кубита $\operatorname{Re} \beta_{1}, \operatorname{Im} \beta_{1}$ плавно зависят от времени (рис. $6, a$ ), тогда как аналогичные величины для второго нерезонансного кубита осциллируют с разностной частотой $\Omega_{2}-\Omega_{1}$ равной $5 \mathrm{MHz}$ (рис. $6, b$ ).

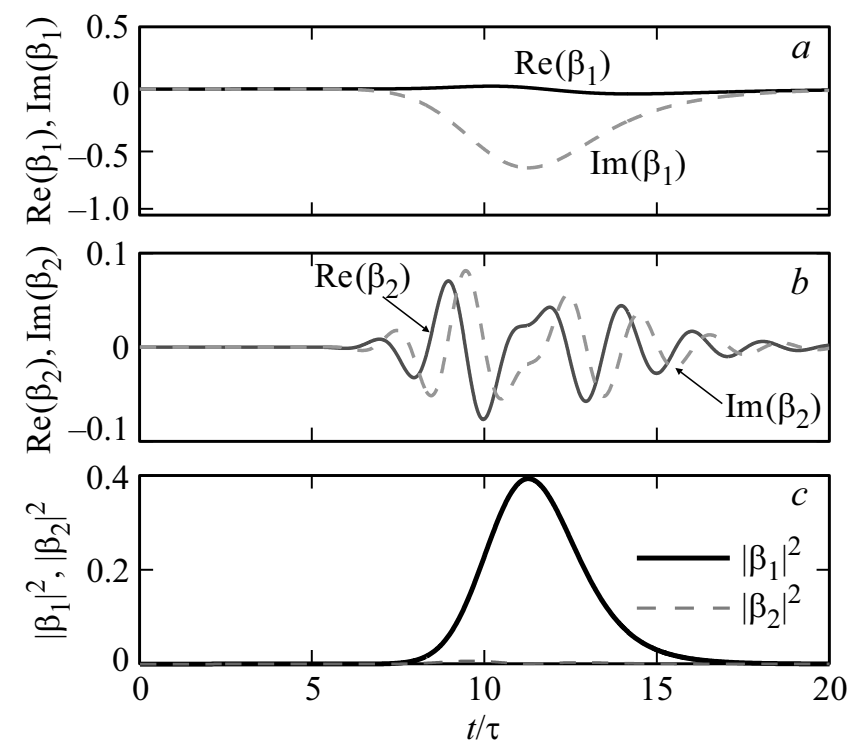

Рис. 6. Возбуждение двух неидентичных кубитов однофотонным импульсом. $k d=0.033 \pi, d=1 \mathrm{~mm}, \Omega_{1} / 2 \pi=\omega_{s} / 2 \pi$ $=5 \mathrm{GHz}, \quad \Omega_{2}=1.001 \Omega_{1}, \quad \Gamma_{1}=\Gamma_{2}=\Gamma, \quad \Gamma / 2 \pi=1.6 \mathrm{MHz}$. $a$ - зависимость от времени вещественной и мнимой части резонансного кубита. $b-$ зависимость от времени вещественной и мнимой части нерезонансного кубита. $c-$ зависимость от времени вероятности возбуждения двух кубитов.

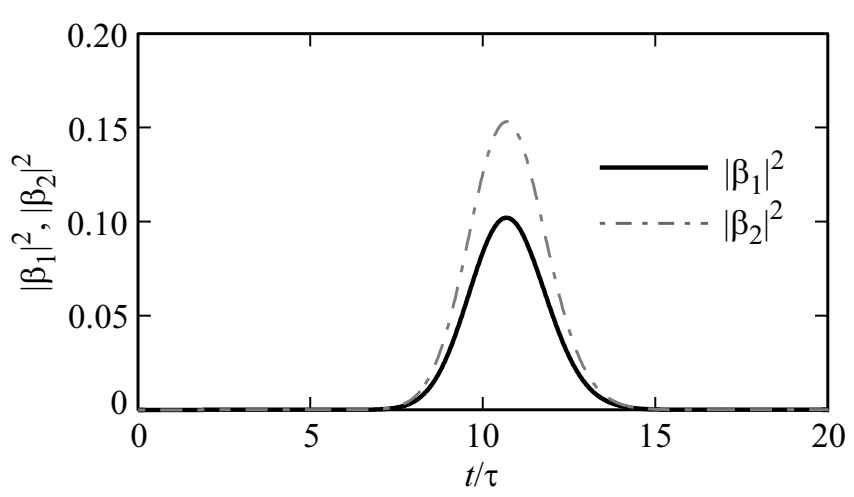

Рис. 7. Вероятность возбуждения двух неидентичных кубитов однофотонным импульсом. $k d=0.033 \pi, d=1 \mathrm{~mm}, \Omega_{1} / 2 \pi=$ $=\omega_{s} / 2 \pi=5 \mathrm{GHz}, \Omega_{2}=\Omega_{1}, \Gamma_{1} / 2 \pi=1.6 \mathrm{MHz}, \Gamma_{12}=1.5 \Gamma_{1}$.

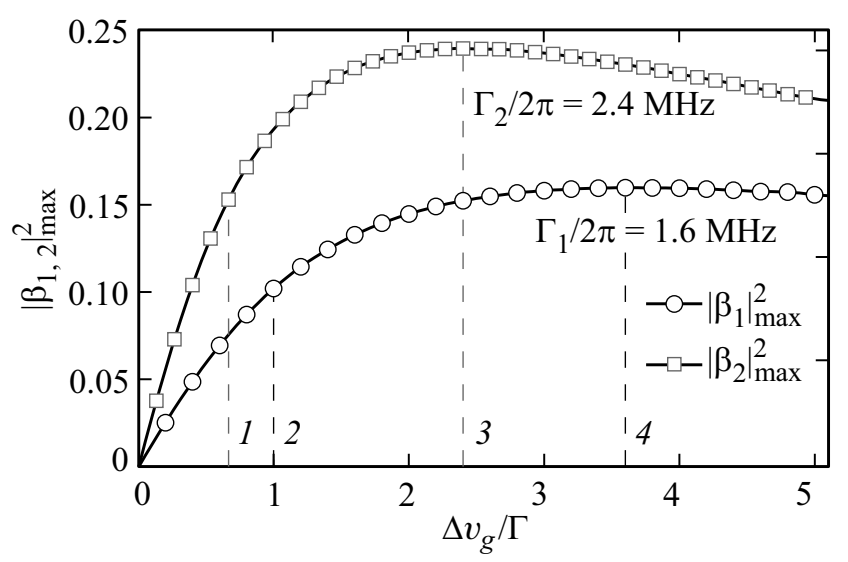

Рис. 8. Зависимость максимальных амплитуд вероятности возбуждения двух неидентичных кубитов от отношения $\quad \Delta v_{g} / \Gamma . \quad k d=0.033 \pi, \quad d=1 \mathrm{~mm}, \quad \Omega_{1} / 2 \pi=\omega_{s} / 2 \pi$ $=5 \mathrm{GHz}, \Omega_{2}=\Omega_{1}, \Gamma_{1} / 2 \pi=1.6 \mathrm{MHz}, \Gamma_{2}=1.5 \Gamma_{1}$. Для каждой кривой расчет проводился при фиксированной величине $\Gamma_{1}$ или $\Gamma_{2}$ соответственно.

На рис. 7 показаны амплитуды возбуждения двух кубитов с одинаковыми энергиями $\left(\Omega_{1}=\Omega_{2}\right)$, но разной скоростью спонтанного излучения $\left(\Gamma_{1} \neq \Gamma_{2}\right)$. Из этого рисунка видно, что амплитуда возбуждения больше у того кубита, у которого скорость спонтанного излучения больше. Это объясняется тем, что величина скорости спонтанного распада непосредственно связана с величиной взаимодействия кубита с электромагнитным полем (см. (7)). Чем больше $g_{k}^{(i)}$, тем больше Г и, следовательно, тем сильнее кубит взаимодействует с полем, что приводит к увеличению вероятности возбуждения. Эта особенность хорошо видна на рис. 8, где показаны зависимости от $\Delta v_{g} / \Gamma$ максимальных амплитуд возбуждения двух кубитов с разными значениями Г. Как видно из этого рисунка, максимальная амплитуда возбуждения кубита с бо́льшим значением скорости спонтанного распада (меньшим значением отношения $\Delta v_{g} / \Gamma$ ) всегда больше аналогичной величины для кубита с меньшим значением скорости спонтанного распада (бо́льшим зна- 
чением отношения $\left.\Delta v_{g} / \Gamma\right)$. Причем, эта особенность не связана с тем, какой кубит возбуждается первым и слабо чувствительна к расстоянию между кубитами. На рис. 8 расстояние между кубитами много меньше длины волны, однако, наши расчеты показывают, что и при $d \gg \lambda$ эта особенность сохраняется: кубит с меньшим значением отношения $\Delta v_{g} / \Gamma$ имеет большую вероятность возбуждения.

\section{4. Заключение}

В работе в марковском приближении исследована динамика возбуждения двух кубитов, находящихся в одномерном волноводе, однофотонным пакетом гауссовой формы. Рассмотрено возбуждение как идентичных, так и неидентичных кубитов. Показано, что при импульсном возбуждении двух одинаковых кубитов возможно формирование симметричных и антисимметричных запутанных состояний. Обнаружено также, что в случае резонансного возбуждения идентичных кубитов фаза амплитуды возбуждения каждого из кубитов не зависит от времени. Показано также, что амплитуды возбуждения двух кубитов с одинаковыми частотами возбуждения зависят от скорости спонтанного распада каждого из кубитов: вероятность возбуждения больше у того кубита, у которого больше скорость спонтанного распада.

Авторы выражают благодарность А.Н. Султанову за полезные обсуждения.

\section{Список литературы}

[1] Y. Wang, J. Minar, L. Sheridan, V. Scarani. Phys. Rev. A 83, 063842 (2011).

[2] M. Stobińska, G. Alber, G. Leuchs. Europhys. Lett. 86, 14007 (2009).

[3] E. Rephaeli, J.-T. Shen, S. Fan. Phys. Rev. A 82, 033804 (2010).

[4] Y. Chen, M. Wubs, J. Mørk, A.F. Koendrink. New J. Phys. 13, 103010 (2011).

[5] P. Domokos, P. Horak, H. Ritsch. Phys. Rev. A 65, 033832 (2002).

[6] S. Derouault, M.A. Bouchene. Phys. Lett. A 376, 3491 (2012).

[7] J.-F. Huang, J.-Q. Liao, C.P. Sun. Phys. Rev. A 87, 023822 (2013).

[8] S. Derouault, M.A. Bouchene. Phys. Rev. A 90, 023828 (2014).

[9] Z. Liao, X. Zeng, Shi-Yao Zhu, M.S. Zubairy. Phys. Rev. A 92, 023806 (2015).

[10] M.O. Scully, M.S. Zubairy. Quantum optics. Cambridge University Press, Cambridge, UK (1997).

[11] Б.И. Иванов, И.Л. Новиков, А.Н. Султанов, Я.С. Гринберг, А.В. Кривецкий, А.Г. Вострецов, Е.В. Ильичев. Письма в ЖЭТФ 103, 475 (2016).

[12] И.Л. Новиков, Б.И. Иванов, А.Н. Султанов, Я.С. Гринберг, Е.В. Ильичев. ФТТ 58, 2085 (2016).

Редактор Ю.Э. Китаев 